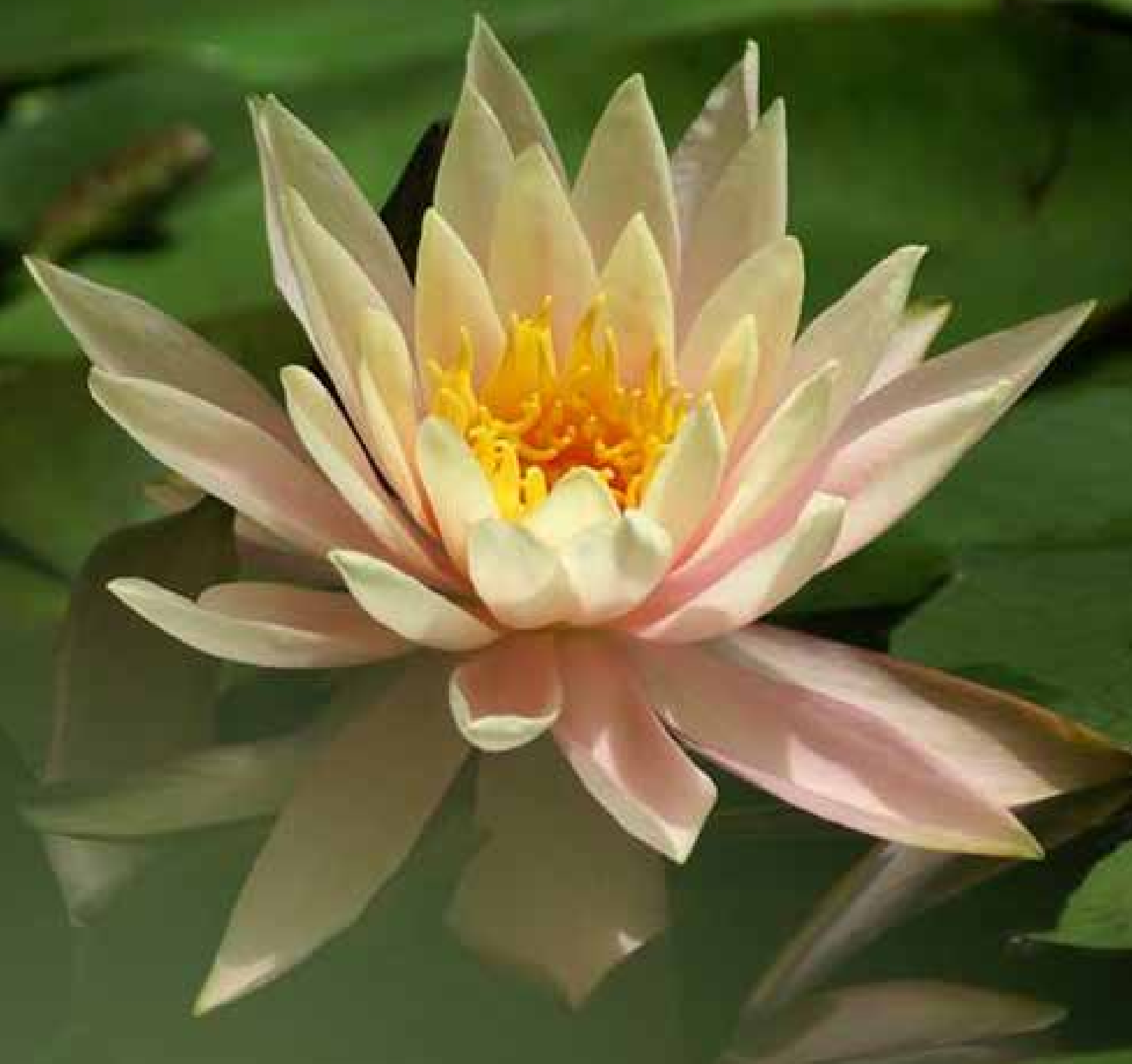




\section{ELEMENTOS MEDULARES DE UNA PROPUESTA DE ENSEÑANZA DE LA GENÉTICA QUE ARTICULE CTSY ALFABETIZACIÓN CIENTÍFICA}

\section{Core Elements of a Teaching Proposal for Genetics that Articulates STS and Scientific Literacy}

\section{Elementos medulares de uma proposta de ensino de genética que articula CTS e alfabetização científica}

\section{Alexander Delgado Suarez* Boris Fernando Candela**}

Fecha de recepción: 7 de octubre de 2018

Fecha de aprobación: 20 de marzo de 2019

\section{Resumen}

En este artículo de investigación sobre la enseñanza de la genética a estudiantes de grado noveno se desarrollan los contenidos medulares que debe tener una propuesta de enseñanza, que articule cтs y la alfabetización científica con el contenido de la enseñanza de la genética. Se busca aportar herramientas que fortalezcan la alfabetización científica en la enseñanza de la genética, y disminuir las brechas que se han generado entre cultura-ciencia-tecnología, a través de las herramientas que brinda la CTS. La metodología del estudio tuvo dos fases: la primera focalizada en el método de análisis de contenido, con el cual se llevó a cabo una lectura sistemática y crítica al conjunto de unidades de muestra y contexto, para informar cómo diseñar la propuesta de enseñanza articuladora de los aspectos sociales con los científicos. La segunda, se centró en la implementación de la propuesta obtenida en la primera fase, donde los estudiantes comenzaron a desarrollar una comprensión del fenómeno de la genética en conjunción con los elementos de naturaleza social que subyacen a dicho contenido. Además, se presentaron los perfiles de los estudiantes que reciben este tipo de propuestas. En conclusión, esta propuesta en educación en ciencias permitió articular de forma sinérgica los aspectos sociales con los naturales a través de una orientación de enseñanza de perspectiva cTs.

Palabras clave: ciencia; tecnología y sociedad (стs); alfabetización científica; diseño curricular; genética; aspectos sociales

* Magister en educación en Ciencias. Docente medio tiempo de la Universidad el Valle, Instituto de Educación y Pedagogía. Orcid: https:// orcid.org/0000-0002-9632-0644

Correo electrónico: alexander.delgado@correounivalle.edu.co

** Magíster en Educación. Profesor de Universidad del Valle (IEP). Orcid: https://orcid.org/0000-0002-5833-1975

Correo electrónico: boris.candela@correounivalle.edu.co 


\section{Abstract}

In this research article on the teaching of genetics to ninth-grade students the core contents that a teaching proposal must have are developed, that articulates sts and scientific literacy with the content of the teaching of genetics. It seeks to provide tools that strengthen scientific literacy in the teaching of genetics and reduce gaps that have arisen between culture-science-technology, through the tools provided by sts. The study methodology had two phases: the first focused on the method of content analysis, which allowed to carry out a systematic and critical reading to the set of sampling units and context, to inform how to design the teaching proposal articulating social aspects with scientific ones. The second focused on the implementation of the proposal obtained in the first phase, where the students began to develop an understanding of the phenomenon of genetics in conjunction with the social nature elements that underlie the content. Additionally, the profiles of the students who receive this type of proposals were presented. In conclusion, this kind of proposal in science education made it possible to articulate the social and natural aspects synergistically through an sTs perspective, teaching orientation.

Keywords: Science Technology and Society (sTs); scientific literacy; curricular design; genetics; social aspects

\section{Resumo}

Neste artigo de pesquisa sobre ensino de genética para alunos da nona série, é desenvolvido o conteúdo principal de uma proposta de ensino, que articula a cтs e a alfabetização científica com o conteúdo do ensino de genética. Procura fornecer ferramentas que fortaleçam a alfabetização científica no ensino de genética e reduzam as lacunas que foram geradas entre cultura-ciência-tecnologia, por meio das ferramentas fornecidas pelo cTs. A metodologia do estudo teve duas fases: a primeira focada no método de análise de conteúdo, com a qual foi realizada uma leitura sistemática e crítica do conjunto de unidades amostrais e do contexto, para informar como elaborar a proposta de ensino articulatório de aspectos sociais com cientistas. A segunda, focada na implementação da proposta obtida na primeira fase, onde os alunos começaram a desenvolver uma compreensão do fenômeno da genética em conjunto com os elementos de natureza social subjacentes ao referido conteúdo. Além disso, foram apresentados os perfis dos alunos que recebem esse tipo de proposta. Concluindo, essa proposta no ensino de ciências possibilitou articular sinergicamente aspectos sociais e naturais por meio de uma orientação de ensino na perspectiva da cTs.

Palavras-chave: Ciência, Tecnologia e Sociedade (cTs); alfabetização científica; desenho curricular; genética; aspectos sociais 


\section{Introducción}

La investigación se fundamenta en las ideas de la corriente de Ciencia, Tecnología y Sociedad (CTS) y la alfabetización científica, y su objetivo es generar una propuesta de enseñanza que articule a través de estas ideas los aspectos naturales de la genética con los elementos sociales que de ella derivan desde lo ético, lo político y lo moral. En este sentido, esta clase de propuesta educativa facilita potenciar habilidades para la vida cotidiana del estudiante tales como: participar en el proceso democrático, reflexivo y crítico que subyace a la toma de decisiones informadas a nivel personal y social, con el fin de dar solución a problemáticas relacionadas con los avances genéticos.

Por otro lado, las metas de la educación en ciencias, de perspectiva disciplinar convencional, se focalizan en suministrarles a los estudiantes la oportunidad de desarrollar de manera progresiva un rango de contenidos conceptuales y procedimentales, que quizás les sirven para comprender algunos fenómenos naturales y artificiales. Sin embargo, dicha orientación curricular suele descuidar las implicaciones controversiales en ámbitos sociales, éticos, morales y políticos que subyacen al fenómeno natural estudiado, en este caso, la genética.

Al respecto, Ayuso y Banet (2002) establecen que las personas poseemos desde edades muy tempranas influencias e informaciones sobre la herencia biológica (medios de comunicación- hogar-escuela-comunidad científica), sin haber sido instruidos como debe ser en estos temas y que, desafortunadamente, no coinciden con lo que se dice en la escuela sobre ellos. Por supuesto, esta situación proporciona al ciudadano del común explicaciones poco precisas entre lo aprendido y lo que suministra la ciencia en relación con muchos fenómenos cotidianos. Por todo esto, se considera que el diseño y la implementación de una lección que representa el fenómeno de la genética requiere tener en cuenta las siguientes cuestiones: ¿Cómo contribuyen los contenidos enseñados a la formación de ciudadanos? y ¿Qué secuencia deben tener dichos contenidos para favorecer un mejor aprendizaje y desarrollo personal y social?

A partir de esta situación se recogen las ideas más controvertidas que subyacen a los contenidos de la ciencia, en especial la genética, las cuales gozan de poco protagonismo desde una enseñanza de perspectiva disciplinar, y con ello se genera una propuesta de enseñanza guiada, donde se vincule de forma sinérgica las potencialidades ofrecidas por los contenidos de la ciencia con las implicaciones sociales que los subyacen (ético, moral, político y económico). Jiménez, Caamaño, Oñorbey Pedrinaci (2007) argumentan que la enseñanza de la biología enfrenta nuevos retos en relación con las investigaciones que son de gran impacto social, tanto en las transformaciones de las condiciones de vida humanas, como en términos de la percepción social.

Por ejemplo, esta perspectiva de enseñanza está relacionada de modo estrecho con situaciones como el proyecto del genoma humano y las aplicaciones de la biotecnología (que incluye clonación e ingeniería genética), donde se tiene como expectativa no solo lograr comprender los productos y procesos de las ciencias, sino que los estudiantes logren internalizar las dimensiones de educación ambiental, educación para la salud y educación para entender los problemas con necesidades personales y sociales (agua, energía, salud, ambiente, alimento, entre otros). De ahí que se requiera establecer las conexiones inextricables entre investigación científica e intereses empresariales, sociales y culturales.

Pero este cometido requiere de investigaciones enfocadas hacia tales articulaciones, una de ellas se configura como el movimiento Ciencia, Tecnología y Sociedad (cTs) el cual busca promover la alfabetización científica de manera que se logre, de acuerdo con Cutcliffe (1990), exponer una interpretación de la ciencia y la tecnología como procesos sociales, articulando estos contenidos conceptuales de la genética con sus aspectos sociales a través de situaciones problema que se lleven al aula de clase.

En este mismo sentido, Yashon y Cummings (2010) diseñaron un currículo donde se introducen la bioética y la ley. Para ello, utilizaron actividades de aprendizaje que representan la genética y su papel emergente como una entidad social y cultural en la vida cotidiana de los estudiantes. Desde luego, estas mediaron la comprensión del fenómeno genético en conjunción con las implicaciones sociales, éticas y políticas acerca de la manipulación genética.

Las anteriores asunciones permiten formular el interrogante que direcciona esta investigación:

¿Cuáles son los elementos medulares de una propuesta de enseñanza que articule a través del enfoque cts los contenidos conceptuales de la genética con los aspectos sociales que de ella derivan desde lo ético, lo político y lo moral?

\section{Marco teórico}

Para el desarrollo de la pregunta de investigación se hace necesario presentar el panorama general orientado a la definición de los conceptos más relevantes sobre los que gira su respuesta, los cuales son: alfabetización científica, Ciencia, tecnología y sociedad (CTS) y genética ya que juntos son los garantes de la renovación y nueva visión de la enseñanza de la ciencia hacia un contexto más social y más humano. 


\section{Alfabetización científica}

El término alfabetización científica es un concepto que nace en el contexto de la guerra fría, "fue empleado por Paul de Hart Hurd como objetivo para la enseñanza de las ciencias en 1958, en un artículo emblemático publicado en la revista Educational Leadership" (Membiela, 2002, p. 35). Pero no solo por la preocupación sociopolítica del momento sino como preocupación de que los estudiantes pudiesen aprender y abrirse a las posibilidades ofrecidas por la ciencia, buscando de alguna manera la formación de los jóvenes con prioridad en ciencia y tecnología y así lograr la especialización de ingenieros y científicos. Lo cual más que un asunto social o cultural, escondía intereses políticos-económicos (Fourez, 2005).

Terminada esta época, dos acontecimientos marcan en definitiva el hito de la alfabetización científica, de un lado con la publicación de un informe de la Royal Society de Londres, donde se proclama la alfabetización científica como objetivo social por alcanzar en las próximas décadas, y hacen el llamado a todas las fuerzas sociales a trabajar por dicho objetivo como una prioridad inminente (Membiela, 2002). De otro lado, Fourez (2005) comenta que, en esta misma época, se publicó un documento por la National Science Teacher Association de los Estados Unidos (NSTA) donde se expuso lo que entendían por alfabetización científica según sus criterios; algunos de ellos son:

- Una persona alfabetizada científica y técnicamente es capaz de: utilizar los conceptos científicos e integrar valores y saberes para adoptar decisiones responsables en la vida corriente.

- Reconocer tanto los límites como la utilidad de las ciencias y las tecnologías en el progreso del bienestar humano.

- Comprender que la sociedad ejerce un control sobre las ciencias y las tecnologías, y, asimismo, que las ciencias y las tecnologías imprimen su sello en la sociedad.

- Reconocer tanto los límites como la utilidad de las ciencias y las tecnologías en el progreso del bienestar humano.

- Conocer los principales conceptos, hipótesis y teorías y ser capaz de aplicarlos.

- Comprender que la producción de saberes científicos depende a la vez de procesos de investigación y de conceptos teóricos.

- Saber reconocer la diferencia entre resultados científicos y opiniones personales.

- Comprender las aplicaciones de las tecnologías y las decisiones implicadas en su utilización.
- Tener una cierta comprensión de la manera en que las ciencias y las tecnologías fueron producidas en la historia.

Podemos notar entonces que los criterios de la alfabetización científica antes mencionados tratan de engranar de alguna manera las diferentes dimensiones de la ciencia; la dimensión histórica de la ciencia y la tecnología (comprender que estas han nacido de una historia humana de la cual forman parte) dándole cabida en el currículo de ciencias. La dimensión epistemológica (comprender cómo se construyen las ciencias en nuestra sociedad y cómo trabajan los científicos). La dimensión comunicativa (comprender que las ciencias y las tecnologías son una manera de entender y construir el mundo, compartida y comunicable). Una dimensión ética (las ciencias son una representación de las posibilidades de nuestro actuar). Una dimensión social (aporta elementos importantes para el desarrollo de la sociedad) y, por último, una dimensión humana (reconocer como fundamento principal que las ciencias y las tecnologías deben siempre estar al servicio de la vida y deben defenderla a toda costa).

Hoy día estos criterios se han transformado en objetivos de la educación en ciencias y son referente de proyectos y de publicaciones por parte de diferentes organismos internacionales y nacionales, el Informe del Programa Internacional para la Evaluación de Estudiantes o Informe PISA por ejemplo, comprende el término alfabetización científica como sinónimo de competencia científica, y la define así: "La capacidad para emplear el conocimiento científico, identificar preguntas y obtener conclusiones basadas en pruebas, con el fin de comprender y ayudar a tomar decisiones sobre el mundo natural y los cambios que la actividad humana produce en él". (OCDE, 2006, p. 7). Lo cual nos lleva a comprender que si un estudiante está alfabetizado científicamente no solo será capaz de responder preguntas relacionadas con la ciencia (algo que se hace mucho en nuestras escuelas en la actualidad) sino que podrá comprender que lo que se haga con el mundo desde las ciencias o cualquier actividad humana tiene una implicación en la sociedad y en él mismo.

Por tanto, la alfabetización científica y tecnológica se entiende como la garante de la formación científica integral del ser humano, una formación para la ciudadanía y no para científicos, es decir, la promoción social de un estudiante crítico, reflexivo y democrático en temas que de la ciencia emanen, para que pueda desenvolverse en la sociedad con un rol participante y no indiferente en noticias de carácter científico (televisivas, de periódicos y de revistas). En otras palabras, hacerse una idea del tema y sus posibles vertientes, que sea capaz de tomar opciones políticas en relación con la ciencia, la salud, el medio 
ambiente y la familia, y que pueda comprender el mundo que le rodea desde las respuestas que le brinda la ciencia, no como verdad única sino como una más de tantas.

\section{Perspectiva CTs (Ciencia, Tecnología y Sociedad)}

La necesidad de interacción de la ciencia con la sociedad ha supuesto un arduo trabajo para diversas disciplinas, sobre todo a partir de los años 60 se han realizado diversos esfuerzos por integrar los estudios sociales de la ciencia y la tecnología, en una perspectiva interdisciplinaria que ha recibido diversas denominaciones Science studies, Ciencia de la ciencia, Cienciología (países socialistas europeos), Science and technology studies, o Science, technology and society. En idioma español se ha acuñado con preferencia la noción de estudios en Ciencia, Tecnología y Sociedad (стS) (Jover, 1999), estos constituyen hoy un vigoroso campo de trabajo donde se trata de entender el fenómeno científico-tecnológico en contexto social, tanto en relación con sus condicionantes sociales como en lo que atañe a sus consecuencias sociales y ambientales (Marino, González, Cerezo, Luján, y Gordillo 2001).

En educación, los estudios cts tratan de comprender los aspectos sociales del fenómeno científico y tecnológico, tanto en lo que respecta a sus condicionantes sociales, como lo relacionado con sus consecuencias sociales y ambientales. Su enfoque general es divalente; por un lado, de carácter crítico (con respecto a las visiones clásica y esencialista de ciencia y tecnología donde ocultan sus dimensiones sociales) y por otro interdisciplinar, donde triangulan disciplinas como la Filosofía, la Historia, la Sociología de la Ciencia y la Tecnología, entre otras. Para González García (1996) la perspectiva cTs en educación se trabaja desde tres líneas de acción: como una materia más en el currículo; ciencia y tecnología a través de la cTs (reconstruir los contenidos de la enseñanza de la ciencia y la tecnología a través de la mirada cTs, con situaciones problemas o roles futuros del estudiante, y sobre ello seleccionar los conocimientos); y cTs como añadido de materias (intercalar los contenidos técnicos y de la ciencia, con los contenidos de la cтs).

Por el lado de la educación secundaria, cts suele considerarse una innovación del currículo escolar que da prioridad a los contenidos actitudinales (cognitivos, afectivos y valorativos) y axiológicos (valores y normas) relacionados con la intervención de la ciencia y la tecnología en la sociedad, con el propósito de formar personas capaces de actuar como ciudadanos responsables que puedan tomar decisiones razonadas y democráticas sobre estos problemas en la sociedad civil. Sin embargo, que este enfoque no supone de ninguna manera desviar la enseñanza de la ciencia como muchas veces se ha querido ver, sino que le da sentido a lo que se aprende para hacerlo funcional dentro del aula, aporta a la formación de ciudadanos y les permite participar de forma democrática en la toma de decisiones, ser más responsables socialmente (formación axiológica) de los problemas del presente y contribuye a evitar rupturas drásticas entre la ciencia y la tecnología, cuyo puente es difícil de definir en el mundo actual (Quintero, 2010).

En este sentido, una propuesta de enseñanza y aprendizaje de la genética enfocada a cts debe abordar problemas sociotécnicos relevantes para los estudiantes; situar estos problemas en contextos específicos; introducir el análisis socio-filosófico, ético, político, económico, en estos problemas; promover el desarrollo de capacidades necesarias para argumentar en torno a la toma de decisiones sobre cuestiones CTs, sus problemas éticos, morales y políticos, lo cual permitirá favorecer la apertura del ámbito escolar al medio social, y que transforme ciudadanos con una cultura científica útil (comprometidos, críticos y proponentes) en su vida diaria.

\section{Metodología}

Con el propósito de darle solución al problema de este estudio se toma la decisión de diseñar una metodología de investigación cualitativa con enfoque interpretativo, la cual está configurada en dos fases:

La primera fase fue realizada bajo el método de análisis de contenido cualitativo a partir de la interpretación de textos (revistas, tesis, libros, artículos, páginas web) asociados de forma directa al problema de la investigación (Krippendorff, 1990). Tal interpretación se lleva a cabo por medio una lectura sistemática, reflexiva, crítica, objetiva, replicable y validada a todo el corpus de información contenida en las unidades de contexto. Así pues, a cada una de estas unidades se le realiza un proceso de codificación abierta y axial (Strauss y Corbin, 2002) con el propósito de ordenar de manera conceptual la información contenida en las fuentes documentales y generar así los elementos medulares para la construcción de la propuesta de enseñanza.

Desde luego, dichos elementos medulares deben articular los aspectos científicos de la genética con los elementos sociales. Estos principios refieren a elementos de la pedagogía, la didáctica, la metodología y la evaluación de la propuesta; se seleccionaron de manera deductiva y se tomaron como referencia los ítems curriculares e instruccionales sobre la elaboración de unidades didácticas propuestos por Sánchez y Valcárcel (1993). Las decisiones curriculares e instruccionales que se toman con base en la teoría generada en esta primera fase, permiten generar 
los elementos medulares de un material de enseñanza estructurado en tres guías: 1) "Selección del sexo"; 2) "Genética y comportamiento"; 3) "Genética y futuro". En efecto, cada una de estas recoge un conjunto de actividades de aprendizaje cuyo fin resulta ser el de brindarle a los estudiantes la oportunidad de integrar el contenido de la genética con los elementos de carácter social.

En cuanto a la segunda fase, se aplicó un estudio de caso piloto instrumental (Stake, 1999), a 22 estudiantes de grado $9^{\circ}$ con edades entre 14 y 15 años, del colegio Claretiano Santa Dorotea de la ciudad de Cali, a fin de comenzar a implementar los elementos medulares de la propuesta de enseñanza en el aula de clase y la viabilidad de esta a través de cTs. Para ello, se puso en escena la primera guía del material instruccional, "Selección del sexo", compuesta de cuatro actividades de aprendizaje ${ }^{1}$, a través de la cual los estudiantes comienzan a desarrollar la integración de los contenidos de la genética con los elementos de naturaleza social.

Conviene subrayar, que la selección del caso estuvo direccionado por un conjunto de criterios, a saber: accesibilidad a los sujetos estudiados; suministro de información pertinente para el estudio; sujetos dispuestos a invertir tiempo en las tareas de indagación, estudiantes que estuvieran cursando noveno grado de la educación básica secundaria formal, cuyo currículo prescribe la enseñanza del contenido de la genética; y profesor con un sistema de conocimiento, creencias y valores fundamentado en la perspectiva de integración de los elementos disciplinares y sociales de las ciencias. Desde luego, estos criterios se encuentran en coherencia con los principales aspectos que configuran la formulación y sustentación del problema.

1 Las actividades que componen esta guía son: a) el caso de los Carter; b) biotecnología en la selección del sexo; c) videos de ciencia y eugenesia; d) historieta de Downtown.

\section{Resultados y análisis}

Si se toma como referencia el problema de investigación que se focaliza en la búsqueda de los elementos medulares de un material de enseñanza donde se articule el contenido disciplinar de la genética con los aspectos sociales que los subyacen, junto con la implementación piloto de una de las secuencias de actividades de aprendizaje que representan el contenido, "Selección del sexo", se generan dos productos. El primero, por razones de la complejidad que subyace a la estructura del material de enseñanza, se presenta a través de una tabla que sintetiza algunos de los elementos que sustentaron la toma de decisiones curriculares e instruccionales, los cuales recogen las acciones del profesor y los estudiantes junto con las metas de aprendizaje por alcanzar (Véase tabla 1). En cuanto al segundo, se genera a partir del análisis cualitativo e interpretativo desde la perspectiva de la teoría fundamentada de Strauss y Corbin (2002).

En este sentido, la información recogida a través de las fuentes documentales aplicadas en el colegio durante la investigación (ej., videos, notas de campo y trabajos de los estudiantes) desde la implementación piloto de las actividades que representan el contenido "Selección del sexo", se sometió a un análisis riguroso, apoyado en la teoría fundamentada de Strauss y Corbin (2002). Esta teoría se caracteriza por estar configurada en tres fases: descripción, reducción de datos y teorización. Así, la primera orientó la forma como se recabaron los datos, la segunda permitió realizar procesos de codificación abierta, selectiva y axial a fin de generar un conjunto de categorías (Véase tabla 2); y la última permitió producir una teoría de carácter naturalístico, que describe y explica el caso del diseño e implementación de una propuesta de enseñanza que articule la genética desde un enfoque cTs con los aspectos de orden social que subyacen a esta. Resulta importante destacar que el proceso de generación de las categorías se llevó a cabo de forma independiente por los autores del artículo, con la intención de darle confiabilidad y veracidad a los resultados (triangulación por autor). 
Tabla 1. Representación sucinta de la secuencia de actividades de aprendizaje que informan la construcción del material de enseñanza.

\begin{tabular}{|c|c|c|c|}
\hline & Selección del sexo & $\begin{array}{c}\text { Genética y } \\
\text { comportamiento }\end{array}$ & Genética y futuro \\
\hline Aprendizajes por alcanzar & $\begin{array}{l}\text { Critica, reflexiona y participa } \\
\text { ante situaciones relacionadas } \\
\text { con los contenidos básicos de } \\
\text { la genética (ADN, cromosomas y } \\
\text { célula) y los aspectos sociales } \\
\text { que de ellos derivan tales como: } \\
\text { discriminaciones, tendencias } \\
\text { machistas, leyes sin coherencia } \\
\text { social. }\end{array}$ & $\begin{array}{l}\text { Critica, reflexiona y participa } \\
\text { ante situaciones relacionadas } \\
\text { con el concepto de genoma, } \\
\text { parentesco genético y síntesis de } \\
\text { proteínas y los aspectos sociales } \\
\text { que de ellos derivan tales como: } \\
\text { enfermedades genéticas y } \\
\text { exclusión laboral. }\end{array}$ & $\begin{array}{l}\text { Critica, reflexiona y participa, } \\
\text { ante situaciones relacionadas } \\
\text { con las técnicas del diagnóstico } \\
\text { prenatal y genético, y los } \\
\text { aspectos de orden social: como la } \\
\text { discriminación, la incertidumbre } \\
\text { familiar, los problemas afectivos } \\
\text { y emocionales, las leyes políticas } \\
\text { y judiciales. }\end{array}$ \\
\hline $\begin{array}{l}\text { Por qué es importante que se } \\
\text { aprenda sobre esto }\end{array}$ & $\begin{array}{l}\text { Con estos aprendizajes los } \\
\text { estudiantes podrán comprender } \\
\text { que la ciencia trabaja en función } \\
\text { de problemas reales de su } \\
\text { contexto social y asimismo } \\
\text { tiene una incidencia positiva y } \\
\text { negativa sobre ellos. }\end{array}$ & $\begin{array}{l}\text { Con estos aprendizajes los } \\
\text { estudiantes podrán reconocer } \\
\text { no solo las posibilidades de la } \\
\text { tecnología para la resolución de } \\
\text { problemas socio técnicos, sino } \\
\text { también sus limitaciones. }\end{array}$ & $\begin{array}{l}\text { Con estos aprendizajes los } \\
\text { estudiantes podrán considerar } \\
\text { las consecuencias de los } \\
\text { impactos tecnológicos en la } \\
\text { sociedad y la cultura. }\end{array}$ \\
\hline Dificultades de aprendizaje & \multicolumn{3}{|c|}{$\begin{array}{l}\text { La genética humana no es un tema central, sino que se propone como un aspecto adicional, además, los } \\
\text { contenidos tienden a ser de tipo científico: causa-efecto, probabilístico, experimental y conceptual. Por } \\
\text { esta razón los estudiantes: 1) tienen unos conocimientos previos muy reducidos sobre estos temas; 2) no } \\
\text { han desarrollado actitudes para relacionar estos contenidos con los aspectos sociales derivados de ellos. }\end{array}$} \\
\hline \multirow[t]{2}{*}{ Estrategias de enseñanza } & $\begin{array}{l}\text { Desarrollar un material } \\
\text { instruccional denominado } \\
\text { Selección del sexo, el cual está } \\
\text { compuesto de } 4 \text { actividades, } \\
\text { en las cuales se encuentran: } \\
\text { situaciones problema, videos, } \\
\text { preguntas y debates. }\end{array}$ & $\begin{array}{l}\text { Desarrollar un material } \\
\text { instruccional denominado } \\
\text { Genética y comportamiento, } \\
\text { el cual está compuesto de } \\
3 \text { actividades, en las cuales } \\
\text { se encuentran: situaciones } \\
\text { problema, videos, preguntas y } \\
\text { debates. }\end{array}$ & $\begin{array}{l}\text { Desarrollar un material } \\
\text { instruccional denominado } \\
\text { Genética y futuro, el cual está } \\
\text { compuesto de } 3 \text { actividades, } \\
\text { en las cuales se encuentran: } \\
\text { situaciones problema, videos, } \\
\text { preguntas y debates. }\end{array}$ \\
\hline & \multicolumn{3}{|c|}{$\begin{array}{l}\text { El aula está organizada en pequeños grupos de discusión, estructura interactiva y trabajo individual, } \\
\text { esta perspectiva de gestión del conocimiento le permitirá al estudiante construir significados y significar } \\
\text { acerca de la genética y los factores sociales, éticos y políticos que de ella derivan. }\end{array}$} \\
\hline Actividades de aprendizaje & $\begin{array}{l}\text { Situación problema: ¿Qué } \\
\text { sucede cuando los padres } \\
\text { desean tener un bebé de un sexo } \\
\text { determinado? } \\
\text { El caso de los Carter } \\
\text { La biotecnología en la selección } \\
\text { del sexo } \\
\text { Ingeniería genética y su } \\
\text { incidencia social } \\
\text { Leyes, ética y moral en las } \\
\text { aplicaciones de la genética; } \\
\text { análisis de historieta } \\
\text { "Downtown" }\end{array}$ & $\begin{array}{l}\text { Situación problema: ¿Son } \\
\text { los genes los responsables de } \\
\text { nuestro comportamiento o es el } \\
\text { ambiente y los factores sociales? } \\
\text { 1. Caso "Una conferencia } \\
\text { importante en suspenso" } \\
\text { 2. El proyecto genoma humano y } \\
\text { enfermedades genéticas } \\
\text { 3. El síndrome de Jacobs (xry) }\end{array}$ & $\begin{array}{l}\text { Situación problema: ¿Es el } \\
\text { diagnóstico prenatal una } \\
\text { solución o un problema? } \\
\text { 1. Caso de "Un bebé al que le } \\
\text { diagnostican PKu" } \\
\text { 2. Las técnicas de diagnóstico } \\
\text { prenatal. } \\
\text { 3. Obra teatral sobre material } \\
\text { instruccional }\end{array}$ \\
\hline
\end{tabular}

Fuente: elaboración propia a partir del análisis de contenido 
Tabla 2. Conjunto de categorías generadas desde los procesos de codificación

\begin{tabular}{|c|c|c|c|c|c|}
\hline \multirow[b]{2}{*}{ Categoría } & \multirow[b]{2}{*}{ Subcategoría } & \multicolumn{4}{|c|}{ Frecuencia de ocurrencia } \\
\hline & & AS1 & GC & $\begin{array}{c}\text { Total por } \\
\text { subcategoría }\end{array}$ & $\begin{array}{c}\text { Total } \\
\text { categoría }\end{array}$ \\
\hline \multirow{2}{*}{$\begin{array}{l}\text { 1. Aspectos positivos de la } \\
\text { ciencia }\end{array}$} & $\begin{array}{l}1.1 \text { prevención de } \\
\text { enfermedades }\end{array}$ & 64 & 10 & 74 & \multirow{2}{*}{101} \\
\hline & 1.2 mejora de la calidad de vida & 17 & 12 & 27 & \\
\hline \multirow{2}{*}{$\begin{array}{l}\text { 2. Aspectos controversiales de } \\
\text { la ciencia }\end{array}$} & 2.1 discriminativos & 15 & 12 & 17 & \multirow[b]{2}{*}{36} \\
\hline & 2.2 éticos y morales & 14 & 15 & 19 & \\
\hline \multirow{2}{*}{$\begin{array}{l}\text { 3. Actitudes hacia los métodos } \\
\text { de la ciencia }\end{array}$} & 3.1 favorables & 28 & 5 & 33 & \multirow[b]{2}{*}{38} \\
\hline & 3.2 desfavorables & 5 & 0 & 5 & \\
\hline \multirow{2}{*}{$\begin{array}{l}\text { 4. Control de los avances de la } \\
\text { ciencia }\end{array}$} & 4.1 sociedad & 28 & 11 & 39 & \multirow[b]{2}{*}{73} \\
\hline & 4.2 estado & 35 & 9 & 34 & \\
\hline
\end{tabular}

Fuente: elaboración propia en el proceso de codificación

Con la intención deliberada de construir la teoría naturalística y con base en la perspectiva de la teoría fundamentada de Strauss y Corbin (2002), se seleccionó una categoría central o medular, la cual fue la de mayor nivel de frecuencia de ocurrencia y abstracción (ej., Aspectos positivos de la ciencia). A esta se articularon de manera semántica las otras tres categorías y se produjeron de esta forma tres generalizaciones naturalísticas, cuyo desarrollo teórico dio origen a una teoría situada que describió el caso estudiado (Véase figura 1).

Figura 1. Producción de las generalizaciones naturalísticas a partir de las relaciones semánticas entre las categorías

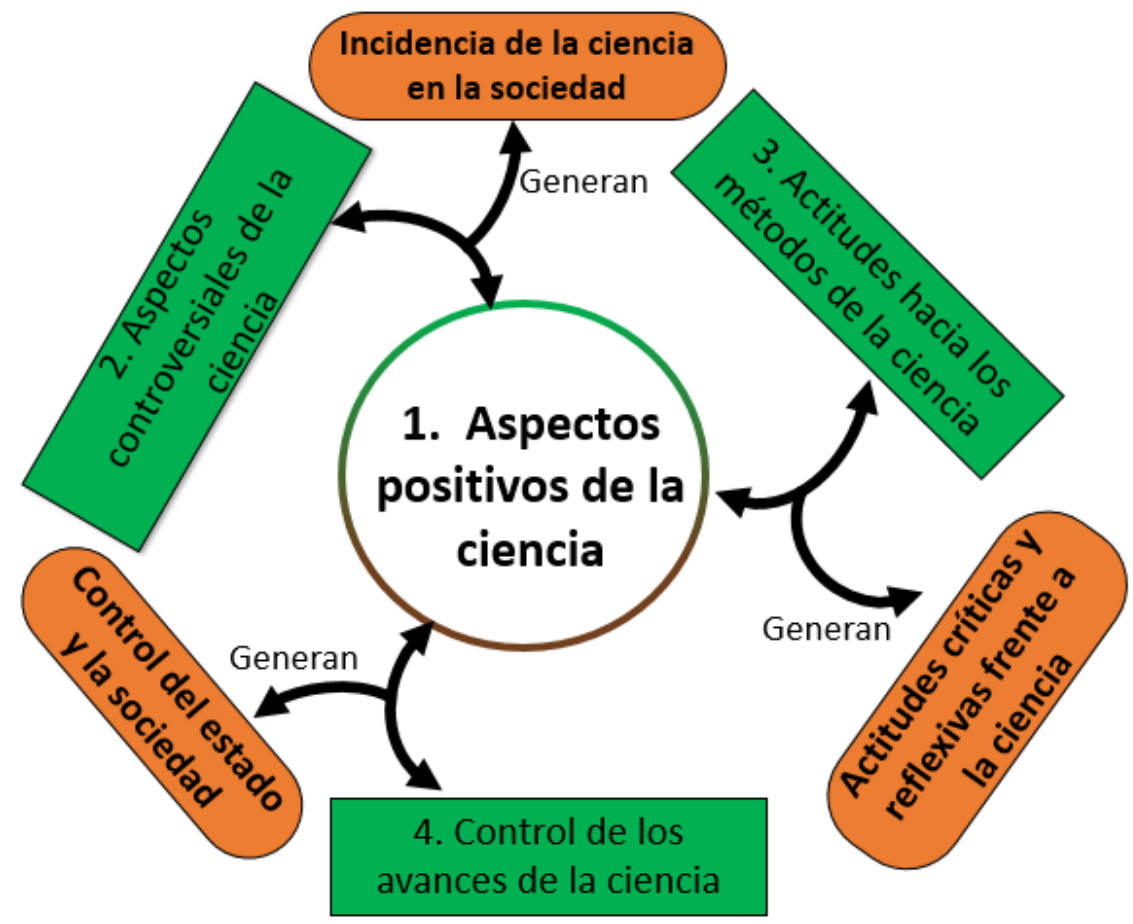

Fuente: elaboración propia 


\section{Presentación de los resultados de la implementación piloto de la actividad Selección del sexo}

El proceso de codificación permite inducir las tres generalizaciones naturalísticas, cuyo desarrollo teórico describe y explica de manera situada la problemática de cómo el diseño e implementación piloto de una propuesta de enseñanza ayuda a los estudiantes a construir la comprensión de la genética, en conjunción con el desarrollo de los aspectos sociales derivados desde lo ético, lo político y lo social. Por supuesto, la teorización de estas tres generalizaciones se encuentra validada a partir de la evidencia proveniente de las diferentes fuentes documentales, resultantes de la implementación piloto de la primera sección del material instruccional denominado: Selección del sexo.

\section{Incidencia de la ciencia en la sociedad}

El conjunto de las cuatro actividades de aprendizaje que representan y formulan el contenido de la guía 1 , "Selección del sexo", se diseña tomando como referencia la información proveniente de la toma de decisiones curriculares e instruccionales que sustentan el material de enseñanza, la cual indica la construcción de una composición textual titulada “El caso de los Carter", en conjunción con un rango de tareas problemas. Desde luego, dichas actividades tienen como propósito central brindar a los estudiantes la oportunidad de comenzar a internalizar las diferentes dimensiones sociales y científicas, relacionadas con los métodos de la selección del sexo. En cuanto a la puesta en escena, la toma de decisiones instruccionales prescribe una organización de aula en pequeños grupos y de discusión con toda la clase, que se encuentra alineada con una orientación de enseñanza centrada en el estudiante.

Por otro lado, la puesta en práctica de las anteriores actividades de aprendizaje se caracteriza por pedirles a los estudiantes que realicen una lectura reflexiva y crítica al texto del caso de los Carter, a fin de dar solución al rango de tareas problemas que acompaña la composición textual. Y, más adelante, los estudiantes en una organización de aula de estructura interactiva socializan las respuestas, con miras a construir una solución a las problemáticas de manera colegiada.

El conjunto de razonamientos y acciones de los estudiantes a lo largo de la transacción de significados les permiten comenzar a evidenciar que la ciencia como una empresa humana no es netamente racional, sino que recibe una fuerte influencia de varios elementos y esferas intelectuales de la cultura en la que se encuentra anidada, por ejemplo, sociales, éticos, políticos, filosóficos, económi- cos, entre otros. Por tanto, ellos intentan comprender que la ciencia no solo está implicada en lo que hagan los científicos a nivel del laboratorio, con miras a lograr un reconocimiento económico y social. Evidencias de estos presupuestos se encuentran en las respuestas dadas al interrogante, ¿Qué implicaciones tiene que los padres puedan decidir el sexo de su bebé? Las cuales se recogen en el siguiente diálogo:

E12: ¿Profe, eso se puede hacer?

E2: ¡Obvio no! No ves que es un caso hipotético, es inventado.

E3: ¡Ay, sí se puede! Yo vi eso en Discovery.

E4: Yo también vi un programa donde hablan de ello.

P: Bueno, ahora hablemos de la segunda pregunta: ¿qué opinan de los procedimientos que se utilizan para seleccionar el sexo de un bebé?

E5: Ja, ja, ja. No profe, yo creíque esa gente estaba dedicada a otras cosas, es que a ellos que les va a interesar eso de los bebés.

E2: Pero vos no ves que hasta los hombres ya pueden volverse mujeres, ¿entonces?

E6: Bueno profesor, yo creo que eso está bien, no ve que a mucha gente le gustaría tener un hijo varón, por ejemplo, pero pues eso es lo que Dios quiera, y si ahora ya la ciencia puede ayudar, me parece que eso ayuda a las familias.

E4: a mítambién me parecen bueno, lo que sícreo es que uno debe informarse bien, porque a mi papá le va a interesar la noticia, no ve que somos tres hermanas (risas).

En cuanto a la segunda actividad, la cual consistía en observar dos videos sobre otras aplicaciones de la ciencia -eugenesia y síndrome de Turner-, el docente motiva a los estudiantes a tomar nota de lo que más les llame la atención del video y de las palabras que no conozcan.

En la socialización de esta actividad, se hace notorio que los estudiantes desconocen o no aceptan que haya científicos que se estén dedicando a este tipo de ciencia, y menos que esta haya tenido tanta relevancia en la historia de la humanidad, sobre todo en la Segunda guerra

2 Para respetar la confidencialidad, en este estudio los símbolos $\mathrm{P}$ y $\mathrm{E}$ tienen el significado de profesor y estudiante, respectivamente. 
mundial, por lo que ven en la ciencia un potencial muy esperanzador - desde el punto de vista de la ayuda que brinda a la sociedad-, pero también temeroso, cuando la ejercen personas con malas intenciones. Estas asunciones se evidencian a partir del siguiente diálogo entre el profesor y los estudiantes, extraído de un video de clase.

P: ¿Alguien conocía acerca de la eugenesia?

E4: No sé, no la conozco, no he escuchado de ella.

E5: No profesor, la verdad eso da hasta miedo, que tal que alguien esté por ahíbuscando la manera de acabar con nosotros los negritos, qué miedo.

E:6 Yo algo había escuchado de eso a la profesora de sociales, cuando vimos la Segunda guerra mundial, pero no sabía que se llamaba así.

E7: Sí, la profesora dijo que también se usaba desde antes, a eso no se debería dedicar la ciencia, ya que daña a las personas.

E5: ¡Uy, no! Uno quéva a estudiartanto para dedicarse a eso.

\section{Controlarlos avances de la ciencia}

En concordancia con el conjunto de actividades de aprendizaje que representan y formulan el contenido, "Selección del sexo", se desarrolló la tercera actividad denominada "Biotecnología en la selección del sexo", en conjunción con un rango de tareas problemas. Estas actividades tienen como propósito central permitir a los estudiantes conocer las leyes y los aspectos políticos derivados de la ciencia. En cuanto a la puesta en escena, la toma de decisiones instruccionales prescribe una organización de aula en parejas y luego en pequeños grupos de discusión con toda la clase, que se encuentra alineada con una orientación de enseñanza centrada en el estudiante.

La puesta en práctica de la anterior actividad de aprendizaje se caracteriza por pedirles a los estudiantes que realicen una lectura reflexiva y crítica de los procedimientos usados por la biotecnología en la selección del sexo, a fin de dar solución al rango de tareas problemas que acompaña la historieta. Luego, los estudiantes en una organización de aula de estructura participativa socializan las respuestas, con miras a construir una solución para las problemáticas de manera colegiada.

El conjunto de consideraciones y acciones de los estudiantes a lo largo de la transacción de significados les permiten, de manera consecuente, generar posturas críticas y ampliar su espectro conceptual sobre el trabajo que realizan los científicos. De hecho, ellos concluyen que el Estado debe monitorear con frecuencia las acciones y trabajos de investigación llevadas a cabo por los científicos, a fin de apoyarlos cuando estos sean beneficiosos o para frenarlos en caso de que estén causando daño a la población. Además, afirman que todos los ciudadanos deben alcanzar una comprensión en esta clase de fenómenos naturales y sociales, con miras a la toma de decisiones personales, locales y nacionales referente a las problemáticas relacionadas con estos contenidos.

En el mismo sentido, los estudiantes logran reconocer dos entes principales que deben encargarse de la vigilancia de los avances científicos, el Estado y la sociedad. Sin embargo, piensan que el Estado es el principal responsable de controlar estos avances, haciendo valer las leyes que ya hay sobre la ciencia o generando otras. Estas presunciones se evidencian en la respuesta que cada estudiante consignó en su guía de trabajo, sobre la pregunta de selección múltiple:

¿De quién consideras debe ser la responsabilidad de regular y controlar este tipo de avances biotecnológicos y por qué?
a. El Estado
b. La sociedad
c. La ciencia

Tabla 3. Respuestas a la pregunta B, de la actividad 4

\begin{tabular}{l|c|c|c} 
& Estado & Sociedad & Ciencia \\
\hline $\begin{array}{l}\text { Cantidad de } \\
\text { respuestas }\end{array}$ & 13 & 7 & 2 \\
\hline
\end{tabular}

Fuente: elaboración propia.

En otro sentido, con esta actividad de aprendizaje los estudiantes empiecen a desarrollar diferentes competencias actitudinales. Desde luego, la discusión a lo largo de las tareas problemas los hace conscientes de que, si bien el Estado debe mantener una regulación a la actividad científica en el país, ellos como ciudadanos científicamente alfabetizados, también tienen que comprender las consecuencias sociales de las decisiones que se toman en el marco de las políticas científicas del país. Esta situación les permite asumir posturas críticas y reflexivas ante los nuevos desarrollos científicos y tecnológicos a fin de tomar decisiones informadas a favor del bienestar personal y social, en otras palabras, se transforman en agentes reguladores de lo que la ciencia produce. La asunción de tales habilidades se evidencia en el siguiente diálogo extraído de un video de la clase: 
P: De acuerdo con este tipo de avances de la ciencia y la tecnología, los referidos a las técnicas de selección del sexo, ¿deberían estos procedimientos estar a la orden de cualquier persona o deberían las políticas del país involucrarse, y cómo?

E2: Yo pienso que deberían involucrarse poniendo leyes y límites en estos procedimientos, porque no se tendría que estar aceptando todos los casos, se debería ser prudente y consciente de cómo son las situaciones de los futuros padres investigando bien el caso.

E3: Deberían involucrarse poniendo una ley donde se vea que la técnica es para una necesidad y no por un capricho elegir el sexo de un bebé.

E4: Sí, porque es necesario que se tome control para prevenir no solo esto sino otras prácticas como la prostitución o cosas así. Y de qué manera pues poniendo condiciones para realizar el debido proceso de esto.

E5: No deberían involucrarse porque la decisión es de cada persona y no creo que se deba prohibir.

E6: Yo creo que deben más bien vigilar que estos no sean dañinos para la sociedad, pero también las personas deberían tener derecho a decidir si los aplican o no.

\section{Actitudes crítica y reflexiva frente a las ciencias}

En concordancia con el conjunto de actividades de aprendizaje que representan y formulan el contenido de la guía 1, "Selección del sexo", se desarrolló la cuarta actividad denominada "Downtown", en conjunción con un rango de tareas problemas. Estas actividades tienen como propósito central que los estudiantes puedan emitir juicios de valor y argumentos críticos frente a las aplicaciones de la ingeniería genética. Respecto a su puesta en escena, la toma de decisiones instruccionales determina un trabajo individual y a continuación una organización de aula en pequeños grupos de discusión con toda la clase, que se encuentra alineada con una orientación de enseñanza centrada en el estudiante.

La implementación de la anterior actividad de aprendizaje se caracteriza por solicitar a los estudiantes que realicen una lectura reflexiva y crítica a una historieta denominada "Downtown", con el fin de dar solución al rango de tareas problemas que acompaña la historieta. Los estudiantes, en una organización de aula de estructura participativa, socializan las respuestas con miras a construir una solución a las problemáticas de manera colegiada.
Antes de iniciar con el desarrollo de este punto, es conveniente aclarar que una actitud supone una predisposición a actuar de cierta manera de acuerdo con unos valores que se llevan implícitos. Por tanto las actitudes se fundamentan en la capacidad para valorar la calidad de la información, para interesarse por el conocimiento y la resolución de los problemas, y para adoptar decisiones autónomas y críticas basadas en criterios científicos (Ferrés, Marbà y Sanmartí, 2015); así, las actitudes estan implícitas en el ser del estudiante, pero a su vez se van puliendo y moldeando a través de la puesta en escena de situaciones en contexto que lo lleven a despertar dicho actuar en función de valores.

En consecuencia, el conjunto de consideraciones y acciones de los estudiantes a lo largo de la transacción de significados les permite generar posturas críticas y ampliar su espectro conceptual sobre el trabajo que realizan los científicos. De igual forma, les brinda la oportunidad de desarrollar conciencia de los beneficios que supone el uso de los avances científicos y de los perjuicios que conlleva el mal uso de estos en su vida cotidiana, poniendo de manifiesto que muchas personas se ven afectadas por los usos inadecuados en la aplicabilidad de los métodos derivados de la ingeniería genética. Asimismo, se notó una postura firme sobre la manera como debe mirarse la ciencia, no como una organización infalible y dueña de la verdad, sino como una manera más de entender el mundo, desde sus métodos, leyes y filosofía. Estos presupuestos se representan en las siguientes respuestas:

D: De acuerdo con los métodos de selección del sexo, ¿estos procedimientos qué tan útiles pueden ser para la sociedad? Muy útiles porque, poco útiles porque, depende porque:

E1: Depende, porque si lo hacen por prevenir enfermedades previene que estas se propaguen, pero si es por satisfacción familiar no sería útil en nada.

E2: Poco útiles porque cuando una mujer queda en embarazo le da igual si es hombre o mujer.

E3: Muy útiles porque ayudan a la prevención de enfermedades y reduciría el número de personas afectadas garantizando una mejor calidad de vida para estas personas.

E4: Yo creo que ellos a veces juegan a creerse "Dios", creen que pueden cambiar las cosas que el ser humano tiene desde su nacimiento.

D: Después de observar la caricatura Downtown, ¿cuál consideras que es el mensaje que se le está enviando desde las biotecnologías para la selección del sexo a las personas que hacen parte de nuestra sociedad y padecen de una enfermedad congénita? 
E1: Que hay una discriminación con las personas que padecen la enfermedad porque les están quitando los derechos a esas personas. Y si aprobaron la ley 1346 del 2009 tiene que ser respetada, y a esas personas hay que darles un trato por igual.

E2: Emiten un mensaje de discriminación porque no están aceptando enfermedades que padecerían las próximas generaciones y aplican el método sin tener en cuenta la ley 1346.

E3: Que en un futuro sus hijos pueden nacer sin estas enfermedades, pero a la vez los está discriminando, porque les están diciendo que estar enfermo está mal, que no deben nacer más personas así, les ofrece una mejor calidad de vida a sus hijos de una manera hipócrita.

Por último, es importante tener en cuenta que el desarrollo de un pensamiento crítico y reflexivo de parte de los estudiantes requiere del docente un esfuerzo de gran tamaño en la generación de la actividad y la pregunta apropiadas, de no ser así, sería difícil lograr el desarrollo de esta habilidad. En otras palabras, se determina que los razonamientos y acciones pedagógicas del profesor juegan un papel clave en el andamiaje de los estudiantes, con el fin de que logren articular de manera consciente el contenido de la genética con los aspectos de orden social y ético que le subyacen.

\section{Conclusiones}

Articular a través de la cts los contenidos conceptuales de la genética con los aspectos sociales que de ella derivan desde lo ético, lo político y lo moral permite alcanzar muchos de los objetivos perseguidos por la alfabetización científica; por ejemplo, la promoción social de un estudiante crítico, reflexivo y democrático en temas que emanen de la ciencia, para que pueda desenvolverse en la sociedad con un rol participante y no indiferente en noticias de carácter científico (ej., televisivas, de periódicos y de revistas). Es decir, que pueda hacerse una idea del tema y sus posibles vertientes, que sea capaz de tomar opciones políticas en relación con la ciencia, la salud, el medio ambiente y la familia, comprender el mundo que le rodea desde las respuestas que le brinda la ciencia, no como verdad única sino como una más entre otras.

En este mismo sentido, se logra establecer un punto de partida básico e importante en cuanto a la elaboración de propuestas con características de cTs, no solo como un proyecto más en la escuela, planteado desde un problema ambiental, sino desde un problema social, puesto que el estudiante no supone encontrarse con este tipo de pro- puestas debido a las características de la ciencia que ha recibido. Esto permite entonces confirmar que es urgente y necesario continuar trabajando con propuestas de este tipo, dado que los resultados son positivos, solo que tales esfuerzos requieren de un trabajo interdisciplinar, para comprender ambos campos, el social y el científico. Sin embargo, con respecto a este último aspecto, esta propuesta se quedó corta, dado que no se involucró ningún docente de áreas como química, física o ciencias sociales en su aplicación, pero reiteramos, es imprescindible un trabajo interdisciplinar que retome este tipo de propuestas.

Con respecto a las tres generalizaciones naturalísticas: aspectos controversiales de la ciencia, actitudes hacia los métodos científicos y control de los avances de la ciencia (Ver figura 1), es conveniente indicar que lograr el desarrollo de estas fue gracias a las características del material instruccional diseñado. Por ejemplo, en este se inicia la clase con una situación problema denominada, "el caso de los Carter", el cual es de vital importancia para lograr el primer aspecto sobre la comprensión de la incidencia de la ciencia en la sociedad. Al mismo tiempo, estos estudios de caso se convierten en el pivote de las clases y direccionan el desarrollo de toda lección. Además, contextualizan el contenido en consideración dentro de un ámbito, y facilitan que los estudiantes puedan comprender la relación existente entre los contenidos y los problemas sociales.

Así mismo, el establecimiento de redes conceptuales (ADN, cromosomas, ingeniería genética, meiosis, síndromes, ética, política, moral) en el material instruccional ayuda en gran medida, ya que se logra evitar la fragmentación de los contenidos y, de esta forma, comenzar a superar la falta de coherencia curricular en los materiales de enseñanza. De hecho, la toma de decisiones curriculares e instruccionales genera una teoría de naturaleza práctica, que orienta tanto el diseño como la implementación del conjunto de actividades de aprendizaje que representan los contenidos y los aspectos sociales y éticos que los subyacen.

Por todo lo dicho, se considera que este trabajo hace un aporte al marco de la educación en ciencias, porque propende por los objetivos que ella misma plantea, es decir, los formulados desde la alfabetización científica. Más aun, cuando involucra en su desarrollo la perspectiva cтs que tanto bien le hace a la educación en ciencias, y por su carácter interdisciplinar al involucrar el área de ciencias sociales en su accionar. Para terminar, se deja a futuras investigaciones que se interesen en este tipo de propuestas, el reto por seguir articulando lo social y lo científico a través de cTs, no solo temas de genética o ambientales, sino también desde la química o la física. 


\section{Referencias}

Ayuso, G. E. y Banet, E. (2002). Alternativas a la enseñanza de la genética en educación media. Enseñanza de las ciencias: revista de investigación y experiencias didácticas, 133-158.

Cutcliffe, S. (1990). Ciencia, tecnología y sociedad: un campo interdisciplinar. En M. Medina y J. Sanmartín (Ed.), Ciencia, tecnología y sociedad, Estudios interdisciplinares en la universidad, en la educación y en la gestión pública (pp. 20-41). Barcelona: Anthropos.

Ferrés, C., Marbà, A. y Sanmartí, N. (2015). Trabajos de indagación de los alumnos: instrumentos de evaluación e identificación de dificultades. Revista Eureka sobre Enseñanza y Divulgación de las Ciencias, 12(1), 22-37. https://revistas.uca.es/index. php/eureka/article/view/2900

Fourez, G. (2005). Alfabetización científica y tecnológica: acerca de las finalidades de la enseñanza de la ciencia. Buenos Aires: Colihue.

González García, M. I. (1996). Ciencia, tecnología y sociedad: una introducción al estudio social de la ciencia y la tecnología. Madrid: Technos.

Jiménez, M. P., Caamaño, A., Oñorbe, A. y Pedrinaci, E. (2007). La enseñanza y el aprendizaje de la biología. En M. P. Jiménez, Enseñar ciencias (pp. 121-146). Barcelona: Grao.

Jover, J. N. (1999). La ciencia y la tecnología como procesos sociales. Lo que la educación científica no debería olvidar. La Habana: Félix Varela.
Krippendorff, K. y Wolfson, L. (1990). Metodología de análisis de contenido: teoría y práctica. Barcelona: Paidós.

Marino, E., González, J. C., Cerezo, J. A., Lujan, J. L. y Gordillo, M. M. (2001). Ciencia, tecnología y sociedad: Una aproximación conceptual. Madrid: oEl.

Membiela, P. (2002). Enseñanza de las ciencias desde la perspectiva Ciencia, Tecnología y Sociedad: formación científica para la ciudadanía. Vol. 89. Madrid: Narcea.

OCDE (2006). PISA, Marco de la evaluación: Conocimientos y habilidades en Ciencias, Matemáticas y lectura. España: Santillana educación.

Quintero, C. A. (2010). Enfoque, Ciencia tecnología y Sociedad: perspectivas para Colombia. Zona próxima, 222-239.

Sánchez, G. y Valcárcel, M. (1993). Diseño de unidades didácticas en el área de las Ciencias Experimentales. Enseñanza de las Ciencias, 11 (1), 33-44.

Stake, R. E. (1998). El caso único. En R. E. Stake, Investigación con estudio de casos. Madrid: Editorial Morata.

Stake, R. (1999). El caso único. Investigación con estudio de casos. Madrid: Morata.

Strauss, A. y Corbin, J. (2002). Bases de la investigación cualitativa. Técnicas y procedimientos para desarrollar la Teoría Fundamentada. Medellín, Colombia: Universidad de Antioquia.

Yashon, R. y Cummings, M. (2010). Genética Humana y Sociedad. México DF: Cengage Learning Editores S.A 